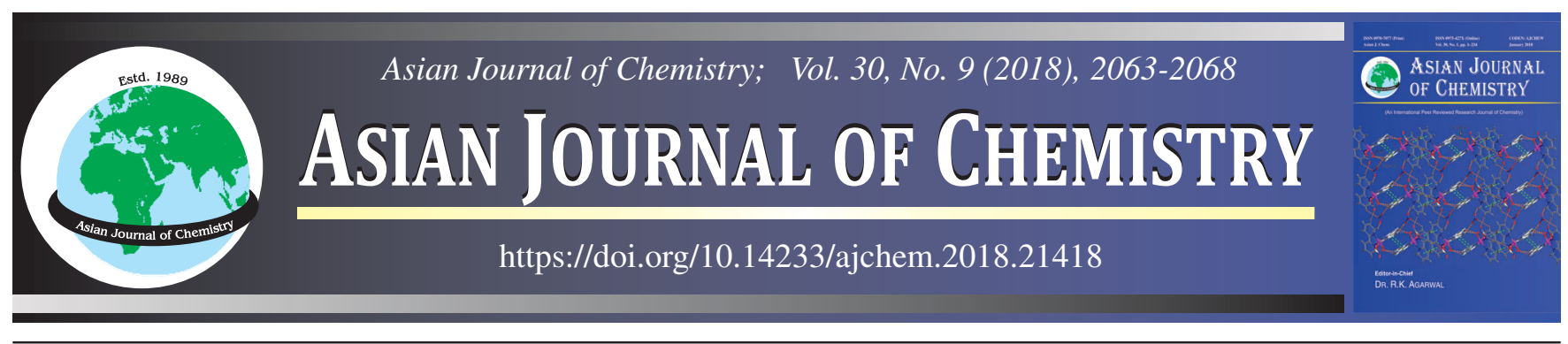

\title{
Synthesis, Characterization and Anticancer Activity of 5-Substituted 4,5,6,7-Tetrahydro- $\mathrm{N}$-(tetrahydro-2H-pyran-4-yl)thieno[3,2-c]pyridine-2-carboxamide Derivatives
}

\author{
N. Sree Lakshmana Rao ${ }^{1}$, Mandava V. Basaveswara RaO ${ }^{2, *}$ and K.R.S. Prasad ${ }^{1}$
}

${ }^{1}$ Department of Chemistry, K.L. University, Vaddeswaram, Guntur-522 502, India

${ }^{2}$ Department of Chemistry, Krishna University, Machilipatnam-521 001, India

*Corresponding author: E-mail: mandavabasaveswararaov@gmail.com

Received: 2 May 2018;

Accepted: 9 June 2018;

Published online: 31 July 2018;

AJC-19021

4,5,6,7-Tetrahydrothieno pyridine (THTP) and their derivatives are an important heterocyclic compounds that exhibits various biological activities viz., antimicrobial activity, antileishmanial activity, antiarrhythmic activity, antiinflammatory activity, antihyperlipidemic activity, antidepressant activity, anticancer activity, antiplatelet activity and antidiabetic etc. The present study describes the synthesis, characterization and in vitro cytotoxic potential against human lung carcinoma of some novel derivatives of 4,5,6,7-tetrahydrothieno[3,2-c]pyridine (7AM) with benzylic and amide substitution on the nitrogen atom of tetrahydro theino pyridine ring. The synthetic steps involves (i) Vilsmeyer protocol in step 1 (ii) formation of tetrahydothieno[3,2-c]pyridine ring in presence of 2-mercaptoacetate in step 2 (iii) alkaline hydrolysis followed by amide coupling with tetrahydro- $2 \mathrm{H}$-pyran-4-amine in step 3 and step 4 . The newly synthesized compounds $7 \mathbf{A}$-M was sufficiently characterized by ${ }^{1} \mathrm{H}$ NMR, IR and mass techniques. Furthermore, these derivatives were screened for their in vitro cytotoxic potential against human lung carcinoma (HCT-116) cell line using the MTT assay. Compound 7B ( $\mathrm{IC}_{50}: 69.52 \mu \mathrm{g}$ ) and compound 7K $\left(\mathrm{IC}_{50}: 66.35 \mu \mathrm{g}\right.$ ) exhibited significant activity at micro molar concentration when compared to standard drug camptothecin.

Keywords: Anticancer activity, Camptothecin, Synthesis, Tetrahydothieno[3,2-c]pyridine, Villsmeyer-Hack.

ᄂ _ - - - - - - - - - - - - - - - - - - - - - - - - - - -

\section{INTRODUCTION}

4,5,6,7-Tetrahydrothieno-pyridine (THTP) represents a widely used lead structure with multitude of interesting applications in numerous pharmacological fields. Thus, various biological activities such as antimicrobial activity [1-15], antileishmanial activity [16], antiarrhythmic activity [17], antiinflammatory activity [18-22], antihyperlipidemic activity [23], antidepressant activity [24], anticancer activity [25,26], antiplatelet activity [27,28], antidiabetic [29-31] and antituberculosis activity $[32,33]$ have been reported and explored till date. Fusion of other nuclei like benzene, indole, oxadiazole, triazole rings to THTP nucleus enhances the pharmacological activities than its parent nucleus.

The incorporation of benzylic or substituted benzylic groups on the nitrogen of the thienopyridine ring can bring an extensive modification in the biological activities of parent compound [34-36]. Hence, different substitutions at nitrogen of the biological activity of the new chemical entities (NCEs). The present study describes the synthesis, characterization and in vitro cytotoxic potential against human lung carcinoma of some novel derivatives of 4,5,6,7-tetrahydrothieno[3,2c]pyridine (7A-M) with benzylic and amide substitution on the nitrogen of the thieno pyridine ring.

\section{EXPERIMENTAL}

The solvents were purified according to standard procedures prior to use and all commercial chemicals were used as received. For thin-layer chromatography (TLC) analysis, Merck pre-coated plates (silica gel 60 F254) were used and eluting solvents are indicated in the procedures. Merck silica gel 60 (230-400 mesh) was used for flash column chromatography. Melting point determinations were performed by using Meltemp apparatus and are uncorrected. ${ }^{1} \mathrm{H}$ NMR spectra were recorded on a Varian Unity instrument at room temperature at $400 \mathrm{MHz}$. Chemical shifts are reported in $\delta$ parts per million (ppm) downfield from tetramethylsilane (TMS) with reference to internal solvent and coupling constants in Hz. The mass spectra were recorded on Agilent ion trap MS. Infrared spectra were recorded on a Perkin Elmer FT-IR spectrometer.

This is an open access journal, and articles are distributed under the terms of the Creative Commons Attribution-NonCommercial 4.0 International (CC BY-NC 4.0) License, which allows others to copy and redistribute the material in any medium or format, remix, transform, and build upon the material, as long as appropriate credit is given and the new creations are licensed under the identical terms. 
tert-Butyl 3-formyl-4-oxopiperidine-1-carboxylate (2): To a stirred solution of tert-butyl 4-oxopiperidine-1-carboxylate (1) (40 g, $0.20 \mathrm{~mol})$ in dichloromethane $(500 \mathrm{~mL})$ was added DMF $(30.9 \mathrm{~mL}, 0.40 \mathrm{~mol})$ followed by $\mathrm{POCl}_{3}(30 \mathrm{~mL}$, $0.32 \mathrm{~mol}$ ) at $0{ }^{\circ} \mathrm{C}$ and the reaction mixture was stirred at room temperature for $4 \mathrm{~h}$. After completion of the reaction (monitored by TLC), the reaction mixture was quenched with aqueous sodium acetate solution (at $0{ }^{\circ} \mathrm{C}$ ), diluted with water and extracted with ethyl acetate $(2 \times 100 \mathrm{~mL})$. The organic layer was washed with water $(3 \times 50 \mathrm{~mL})$, brine solution, dried over anhydrous sodium sulfate, filtered and evaporated under reduced pressure to obtain compound 2 . The crude compound was used in the next step without any purification. Yellow oil; Yield: $30 \mathrm{~g}, 65 \%$; Elemental analysis, $\mathrm{C}_{11} \mathrm{H}_{17} \mathrm{NO}_{4}$, calcd. (found) \%: C 58.14 (58.11), H 7.54 (7.56), N 6.16 (6.12). IR (KBr, $\left.v_{\max }, \mathrm{cm}^{-1}\right): 2926(-\mathrm{CH}$ str. ), 1695, 1635 (-C=O str.), 1122 (-C-OC- str.); ${ }^{1} \mathrm{H}$ NMR $(500$ $\left.\mathrm{MHz}, \mathrm{CDCl}_{3}\right) \delta: 10.14(\mathrm{~s}, 1 \mathrm{H}), 4.15(\mathrm{~d}, J=7.0 \mathrm{~Hz}, 2 \mathrm{H}), 3.72$ (t, $J=6.0 \mathrm{~Hz}, 2 \mathrm{H}), 2.44$ (t, $J=6.0 \mathrm{~Hz}, 2 \mathrm{H}), 2.09$ (brs, 2H), 1.45 (s, 9H); MS: calcd. for $\mathrm{C}_{15} \mathrm{H}_{21} \mathrm{NO}_{4} \mathrm{~S}[\mathrm{~m} / \mathrm{z}] 311.40$; found: $312.6[\mathrm{M}+\mathrm{H}]^{+}$.

5-tert-Butyl 2-ethyl-6,7-dihydrothieno[3,2-c]pyridine2,5-(4H)-dicarboxylate (3): To a stirred solution of compound $2(30 \mathrm{~g}, 0.132 \mathrm{~mol})$ in dichloromethane $(150 \mathrm{~mL})$ was added triethyl amine $(29.1 \mathrm{~mL}, 0.211 \mathrm{~mol})$ and ethyl 2-mercaptoacetate $(17.3 \mathrm{~mL}, 0.158 \mathrm{~mol})$ at $0{ }^{\circ} \mathrm{C}$. The reaction mixture was stirred at $45^{\circ} \mathrm{C}$ for $16 \mathrm{~h}$. After completion of reaction, the reaction mixture was diluted with ice cold water and extracted with dichloromethane $(3 \times 50 \mathrm{~mL})$. The organic layer was washed with water, brine solution, dried over anhydrous sodium sulphate, filtered and evaporated under reduced pressure to afford compound 3 . The crude compound ( $30 \mathrm{~g}$ ) was used in the next step without any further purification. Elemental analysis, $\mathrm{C}_{15} \mathrm{H}_{21} \mathrm{NO}_{4} \mathrm{~S}$, calcd. (found) \%: C 57.86 (57.84), H 6.80 (6.77), N 4.50 (4.53).

5-(tert-Butoxycarbonyl)-4,5,6,7-tetrahydrothieno[3,2c]pyridine-2-carboxylic acid (4): To a stirred solution of compound $\mathbf{3}(30 \mathrm{~g}, 0.096 \mathrm{~mol})$ in a mixture of tetrahydrofuran, methanol and water $(250 \mathrm{~mL}, 3: 1: 1)$ was added sodium hydroxide $(11.5 \mathrm{~g}, 0.288 \mathrm{~mol})$ and stirred at room temperature for $16 \mathrm{~h}$. After completion of reaction, the reaction mixture was evaporated under reduced pressure to obtain a yellow viscous residue. The residue was acidified with aqueous citric acid solution to about $\mathrm{pH} 4$ and the resulting mixture was stirred at $0{ }^{\circ} \mathrm{C}$ for $15 \mathrm{~min}$. The obtained solid was filtered, washed with water and dried under vacuum to afford compound 4. Yellow solid; Yield: 15 g, $55 \%$; m.p.: 179- $181{ }^{\circ} \mathrm{C}$; Elemental analysis, $\mathrm{C}_{13} \mathrm{H}_{17} \mathrm{NO}_{4} \mathrm{~S}$, calcd. (found) \%: C 55.11 (55.15), H 6.05 (6.09), $\mathrm{N} 4.94$ (4.97). IR (KBr, $\left.v_{\max }, \mathrm{cm}^{-1}\right): 3598,3492,2982,2441$, 1658, 1560, 1465, 1416, 1256, 1156, 1012, 947, 856, 759, 601,

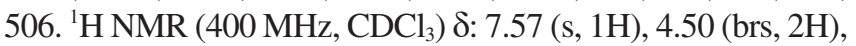
3.73 (d, $J=6.0 \mathrm{~Hz}, 2 \mathrm{H}$ ), 2.89 (brs, 2H), 1.49 (s, 9H); MS: calcd. for $\mathrm{C}_{13} \mathrm{H}_{17} \mathrm{NO}_{4} \mathrm{~S}$ [m/z] 283.34; found: $282.21[\mathrm{M}-\mathrm{H}]^{+}$.

tert-Butyl 2-(tetrahydro-2H-pyran-4-ylcarbamoyl)6,7-dihydrothieno[3,2-c]pyridine-5(4H)-carboxylate (5): The a stirred mixture of compound 4 (8 g, $28.26 \mathrm{mmol})$ in DMF $(80 \mathrm{~mL})$ was sequentially added $\mathrm{EDC} \cdot \mathrm{HCl}(8.2 \mathrm{~g}, 42.3$ mmol), HOBt (4.5 g, $33.8 \mathrm{mmol})$, diisopropylethylamine (14.7 $\mathrm{mL}, 84.6 \mathrm{mmol}$ ) and tetrahydro- $2 \mathrm{H}$-pyran-4-amine (4.2 g, 31.0 $\mathrm{mmol}$ ) at room temperature stirred for $5 \mathrm{~h}$. After completion of reaction, the reaction mixture was quenched with water and extracted with ethyl acetate $(4 \times 25 \mathrm{~mL})$. The organic layer was washed with water, brine solution, dried over anhydrous sodium sulfate, filtered and evaporated under reduced pressure to afford compound 5. White solid; Yield: 5 g, $77 \%$; m.p.: 146$149{ }^{\circ} \mathrm{C}$. Elemental analysis, $\mathrm{C}_{18} \mathrm{H}_{26} \mathrm{~N}_{2} \mathrm{O}_{4} \mathrm{~S}$, calcd. (found) $\%$ : C 58.99 (58.96), H 7.15 (7.12), N 7.64 (7.62). IR (KBr, $v_{\max }$, $\left.\mathrm{cm}^{-1}\right): 3274,2964,2845,2360,1696,1616,1539,1402,1244$, 1164, 1093, 869, 777, 661; ${ }^{1} \mathrm{H}$ NMR (400 MHz, DMSO- $\left.d_{6}\right) \delta$ : $8.21(\mathrm{~d}, J=6.4 \mathrm{~Hz}, 1 \mathrm{H}), 7.53(\mathrm{~s}, 1 \mathrm{H}), 4.40$ (s, 1H), 3.94-3.85 $(\mathrm{m}, 3 \mathrm{H}), 3.63(\mathrm{t}, J=5.6 \mathrm{~Hz}, 2 \mathrm{H}), 3.38-3.33(\mathrm{~m}, 2 \mathrm{H}), 2.78(\mathrm{~s}, 2 \mathrm{H})$, 1.74-1.70 (m, 2H), 1.58-1.54 (m, 4H), 1.42 (s, 9H); ${ }^{13} \mathrm{C} \mathrm{NMR}$ $\left(100 \mathrm{MHz}, \mathrm{DMSO}-d_{6}\right): \delta 160.3,153.9,137.9,137.3,132.9,125.9$, 79.2, 66.0 (2C), 45.6 (2C), 43.5 (2C), 32.4 (2C), 28.0 (4C); MS: calcd. for $\mathrm{C}_{18} \mathrm{H}_{26} \mathrm{~N}_{2} \mathrm{O}_{4} \mathrm{~S}[\mathrm{~m} / \mathrm{z}] 366.48$; found: $367.30[\mathrm{M}+\mathrm{H}]^{+}$.

N-(Tetrahydro-2H-pyran-4-yl)-4,5,6,7-Tetrahydrothieno[3,2-c]pyridine-2-carboxamide.TFA salt (6): To a stirred solution of compound 5 ( $2 \mathrm{~g}, 5.46 \mathrm{mmol})$ in DCM (25 $\mathrm{mL})$ was added trifluoroacetic acid $(0.8 \mathrm{~mL}, 10.0 \mathrm{mmol})$ and the reaction mixture was stirred at $0{ }^{\circ} \mathrm{C}$ for $16 \mathrm{~h}$. After completion of reaction, the reaction mixture was evaporated under reduced pressure to afford compound 6. White solid; Yield: $1.2 \mathrm{~g}, 77 \%$; m.p.: 194-197 ${ }^{\circ} \mathrm{C}$; Elemental analysis, $\mathrm{C}_{13} \mathrm{H}_{18} \mathrm{~N}_{2} \mathrm{O}_{2} \mathrm{~S}$, calcd. (found) \%: C 58.62 (58.60), H 6.81 (6.78), N 10.52 (10.54). IR $\left(\mathrm{KBr}, v_{\max }, \mathrm{cm}^{-1}\right): 3295,2991,2839,2360,1687,1623,1687$, 1623, 1538, 1204, 1145, 836, 792, 719; ${ }^{1} \mathrm{H}$ NMR (400 MHz, DMSO-d ) $\delta: 9.06$ (brs, $1 \mathrm{H}), 8.33$ (d, $J=7.6 \mathrm{~Hz}, 1 \mathrm{H}), 7.56$ (s, $1 \mathrm{H}), 4.20$ (brs, 2H), 3.93-3.84 (m, 3H), 3.0 (t, $J=5.6 \mathrm{~Hz}, 2 \mathrm{H})$, 1.74-1.71 (m, 2H), 1.58-1.49 (m, 2H); ${ }^{13} \mathrm{C} \mathrm{NMR} \mathrm{(100} \mathrm{MHz,}$ DMSO- $\left.d_{6}\right): \delta 160.1,138.48,136.42,128.84,126.0,66.1,45.81$, 41.97, 40.75, 32.42, 21.75. MS: calcd. for $\mathrm{C}_{15} \mathrm{H}_{18} \mathrm{~F}_{3} \mathrm{~N}_{2} \mathrm{O}_{3} \mathrm{~S}[\mathrm{~m} / \mathrm{z}]$ 363.38; found: 267.0 [M-TFA $]^{+}$.

General experimental procedure for the synthesis of 5-substituted 4,5,6,7-tetrahydro-N-(tetrahydro-2H-pyran4-yl)thieno[3,2-c]pyridine-2-carboxamide derivatives (7A7M): To a stirred solution of compound $\mathbf{6}(150 \mathrm{mg}, 0.57 \mathrm{mmol})$ in DMF ( $5 \mathrm{~mL})$ was added $\mathrm{K}_{2} \mathrm{CO}_{3}(0.23 \mathrm{~g}, 1.7 \mathrm{mmol})$ followed by respective aryl/hetero aryl halides $(0.08 \mathrm{~mL}, 0.67 \mathrm{mmol})$. The reaction mixture was stirred at $0{ }^{\circ} \mathrm{C}$ for $15 \mathrm{~min}$ and later to room temperature for $6 \mathrm{~h}$. The progress of the reaction was monitored by TLC. After completion of reaction, the reaction mixture was quenched with water $(10 \mathrm{~mL})$ and extracted with ethyl acetate $(2 \times 10 \mathrm{~mL})$. The organic layer was washed with water, brine solution, dried over anhydrous sodium sulfate, filtered and evaporated under reduced pressure to produce compound 7A-M. Yields of the compounds vary between 74$92 \%$.

5-Benzyl-4,5,6,7-tetrahydro-N-(tetrahydro-2H-pyran4-yl)thieno[3,2-c]pyridine-2-carboxamide (7A): White solid; Yield: $135 \mathrm{mg}, 92 \%$; m.p.: $181-183{ }^{\circ} \mathrm{C}$; Elemental analysis, $\mathrm{C}_{20} \mathrm{H}_{24} \mathrm{~N}_{2} \mathrm{O}_{2} \mathrm{~S}$, calcd. (found) \%: C 67.38 (67.36), H 6.79 (6.77), N 7.86 (7.85). IR (KBr, $\left.v_{\max }, \mathrm{cm}^{-1}\right): 3445,3209,3053,2966$, 2925, 2843, 2791, 1695, 1607, 1542, 1460, 1331, 1305, 1275, 1242, 1142, 1086, 1054, 1008, 885, 745, 706, 605, 570; ${ }^{1} \mathrm{H} \mathrm{NMR}$ (400 MHz, DMSO- $\left.d_{6}\right) \delta: 7.37-7.20(\mathrm{~m}, 5 \mathrm{H}), 7.12(\mathrm{~s}, 1 \mathrm{H}), 5.65$ $(\mathrm{d}, J=8.0 \mathrm{~Hz}, 1 \mathrm{H}), 4.17-4.16(\mathrm{~m}, 1 \mathrm{H}), 3.98(\mathrm{t}, J=2.0 \mathrm{~Hz}, 2 \mathrm{H})$, 3.70 (s, 2H), 3.54-3.47 (m, 4H), 2.88 (d, $J=4.8 \mathrm{~Hz}, 2 \mathrm{H}), 2.81$ $(\mathrm{t}, J=4.5 \mathrm{~Hz}, 2 \mathrm{H}), 1.99-1.95(\mathrm{~m}, 2 \mathrm{H}) ;{ }^{13} \mathrm{C} \mathrm{NMR}(100 \mathrm{MHz}$, 
DMSO- $\left.d_{6}\right): \delta 161.1,141.6,137.7,137.0,135.9,135.6,128.9$ (2C), 64.0 (2C), 60.0, 55.1, 52.8, 51.9, 36.6 (2C), 24.5; MS: calcd. for $\mathrm{C}_{20} \mathrm{H}_{24} \mathrm{~N}_{2} \mathrm{O}_{2} \mathrm{~S}$ [m/z] 356.48; found: $357.41[\mathrm{M}+\mathrm{H}]^{+}$.

5-(2-Chlorobenzyl)-4,5,6,7-tetrahydro- $\mathrm{N}$-(tetrahydro2H-pyran-4-yl)thieno[3,2-c]pyridine-2-carboxamide (7B): Light brown solid; Yield: $139 \mathrm{mg}, 86 \%$; m.p.: $153-155{ }^{\circ} \mathrm{C}$; Elemental analysis, $\mathrm{C}_{20} \mathrm{H}_{23} \mathrm{~N}_{2} \mathrm{O}_{2} \mathrm{SCl}$, calcd. (found) \%: $\mathrm{C} 61.45$ (61.46), H 5.93 (5.91), N 7.17 (7.15). IR ( $\left.\mathrm{KBr}, v_{\max }, \mathrm{cm}^{-1}\right)$ : 3280, 3065, 2949, 2924, 2846, 1614, 1571, 1535, 1466, 1440, 1327, 1245, 1135, 1090, 1047, 874, 798, 747, 657; ${ }^{1} \mathrm{H}$ NMR $\left(400 \mathrm{MHz}, \mathrm{DMSO}-d_{6}\right) \delta: 8.15(\mathrm{~d}, J=7.6 \mathrm{~Hz}, 1 \mathrm{H}), 7.53(\mathrm{~d}, J=$ $6.8 \mathrm{~Hz}, 1 \mathrm{H}), 7.45(\mathrm{~d}, J=8.4 \mathrm{~Hz}, 1 \mathrm{H}), 7.36(\mathrm{~s}, 1 \mathrm{H}), 7.33(\mathrm{~d}, J$ $=8.0 \mathrm{~Hz}, 1 \mathrm{H}), 7.23(\mathrm{~d}, J=7.2 \mathrm{~Hz}, 1 \mathrm{H}), 3.92-3.84(\mathrm{~m}, 2 \mathrm{H}), 3.77$ (s, 2H), 3.54 (s, 2H), 3.38-3.33 (m, 3H), 2.82-2.78 (m, 4H), 1.72 (t, $J=10.8 \mathrm{~Hz}, 2 \mathrm{H}), 1.57-1.47(\mathrm{~m}, 2 \mathrm{H}) ;{ }^{13} \mathrm{C}$ NMR $(100 \mathrm{MHz}$, DMSO- $\left.d_{6}\right): \delta 160.4,138.1,136.8,135.7,134.5,133.2,130.6$, 129.2, 128.6, 127, 126.2, 66.1 (2C), 57.5, 52.1, 49.6, 45.6, 32.4 (2C), 25.1; MS: calcd. for $\mathrm{C}_{20} \mathrm{H}_{23} \mathrm{~N}_{2} \mathrm{O}_{2} \mathrm{SCl}[\mathrm{m} / z] 390.93$; found: $391.76[\mathrm{M}+\mathrm{H}]^{+}$

5-(2-Fluorobenzyl)-4,5,6,7-tetrahydro-N-(tetrahydro2H-pyran-4-yl)thieno[3,2-c]pyridine-2-carboxamide (7C): Off white solid; Yield: $140 \mathrm{mg}, 90 \%$; m.p.: $139-141^{\circ} \mathrm{C}$. Elemental analysis, $\mathrm{C}_{20} \mathrm{H}_{23} \mathrm{~N}_{2} \mathrm{O}_{2} \mathrm{SF}$, calcd. (found) \%: C 64.15 (64.13), H 6.19 (6.20), N 7.48 (7.46). IR (KBr, $\left.v_{\max }, \mathrm{cm}^{-1}\right)$ : 3429, 3266, 2948, 2847, 1631, 1541, 1448, 1383, 1309, 1237, 1139, 1079, $878,770,605 ;{ }^{1} \mathrm{H}$ NMR $\left(400 \mathrm{MHz}\right.$, DMSO- $\left.d_{6}\right) \delta: 8.40(\mathrm{~d}, J=$ $7.6 \mathrm{~Hz}, 1 \mathrm{H}), 7.68-7.61$ (m, 3H), 7.42-7.34 (m, 2H), 4.90 (d, $J$ $=13.2 \mathrm{~Hz}, 2 \mathrm{H}), 4.63(\mathrm{~d}, J=13.2 \mathrm{~Hz}, 2 \mathrm{H}), 4.33(\mathrm{~s}, 2 \mathrm{H}), 3.95-$ $3.80(\mathrm{~m}, 6 \mathrm{H}), 3.40-3.28(\mathrm{~m}, 1 \mathrm{H}), 1.72(\mathrm{t}, J=10.4 \mathrm{~Hz}, 2 \mathrm{H})$, $1.59-1.53(\mathrm{~m}, 2 \mathrm{H}) ;{ }^{13} \mathrm{C}$ NMR (100 MHz, DMSO- $\left.d_{6}\right): \delta 161.4$, $161.1,141.6,137.7,137.0,135.9,130.5,128.9,125.4,124.1$, 115.2, 64.0 (2C), 51.9, 55.1, 52.8, 49.2, 36.6 (2C), 24.5; MS: calcd. for $\mathrm{C}_{20} \mathrm{H}_{23} \mathrm{~N}_{2} \mathrm{O}_{2} \mathrm{SF}[\mathrm{m} / \mathrm{z}]$ 374.47; found: $375.37[\mathrm{M}+\mathrm{H}]^{+}$.

5-(3-Fluorobenzyl)-4,5,6,7-tetrahydro-N-(tetrahydro2H-pyran-4-yl)thieno[3,2-c]pyridine-2-carboxamide (7D): Brown solid; Yield: $145 \mathrm{mg}, 92 \%$; Elemental analysis, $\mathrm{C}_{20} \mathrm{H}_{23} \mathrm{~N}_{2} \mathrm{O}_{2} \mathrm{SF}$, calcd. (found) \%: $\mathrm{C} 64.12$ (64.10), H 6.16 (6.17), N 7.46 (7.44); IR (KBr, $\left.v_{\max }, \mathrm{cm}^{-1}\right)$ : 3248, 2952, 2923, 2224, 1613, 1535, 1447, 1331, 1137, 1088, 1011, 761; ${ }^{1} \mathrm{H}$ NMR (400 MHz, DMSO- $\left.d_{6}\right): \delta: 8.12(\mathrm{~d}, J=8.0 \mathrm{~Hz}, 1 \mathrm{H}), 7.46(\mathrm{t}, J=7.6$ $\mathrm{Hz}, 2 \mathrm{H}), 7.33$ (t, $J=7.2 \mathrm{~Hz}, 1 \mathrm{H}), 7.19(\mathrm{t}, J=7.2 \mathrm{~Hz}, 2 \mathrm{H}), 3.87$ $(\mathrm{t}, J=10.4 \mathrm{~Hz}, 4 \mathrm{H}), 3.73(\mathrm{~s}, 2 \mathrm{H}), 3.48-3.44(\mathrm{~m}, 2 \mathrm{H}), 2.79(\mathrm{~d}$, $J=5.2 \mathrm{~Hz}, 2 \mathrm{H}), 2.74(\mathrm{t}, J=4.8 \mathrm{~Hz}, 2 \mathrm{H}), 1.71(\mathrm{~d}, J=11.2 \mathrm{~Hz}$, $2 \mathrm{H}), 1.56-1.49(\mathrm{~m}, 2 \mathrm{H}) ;{ }^{13} \mathrm{C}$ NMR (100 MHz, DMSO- $\left.d_{6}\right): \delta$ 162.56, 161.17, 141.6, 137.7, 136.9, 135.9, 130.5, 128.9, 125.4, 124.1, 115.2, 65.5 (2C), 52.0, 55.7, 53.1, 50.1, 36.8 (2C), 24.2; MS: calcd. for $\mathrm{C}_{20} \mathrm{H}_{23} \mathrm{~N}_{2} \mathrm{O}_{2} \mathrm{SF}$ [m/z] 374.14; found: 374.47 $[\mathrm{M}+\mathrm{H}]^{+}$.

5-(2-Cyanobenzyl)-N-(tetrahydro-2H-pyran-4-yl)4,5,6,7-tetrahydrothieno[3,2-c]pyridine-2-carboxamide (7E): Off white solid; Yield: $145 \mathrm{mg}, 92 \%$; m.p.: $142-144{ }^{\circ} \mathrm{C}$; Elemental analysis, $\mathrm{C}_{21} \mathrm{H}_{23} \mathrm{~N}_{3} \mathrm{O}_{2} \mathrm{~S}$, calcd. (found) \%: $\mathrm{C} 66.12$ (66.09), H 6.08 (6.10), N 11.01 (11.04). IR ( $\left.\mathrm{KBr}, v_{\max }, \mathrm{cm}^{-1}\right)$ : 3248, 2952, 2923, 2224, 1613, 1535, 1447, 1331, 1137, 1088, 1011,$761 ;{ }^{1} \mathrm{H}$ NMR (400 MHz, DMSO- $\left.d_{6}\right): \delta: 8.14$ (d, $J=7.6$ $\mathrm{Hz}, 1 \mathrm{H}), 7.83(\mathrm{~d}, J=8 \mathrm{~Hz}, 1 \mathrm{H}), 7.71-7.61(\mathrm{~m}, 1 \mathrm{H}), 7.63(\mathrm{~d}, J=$ $7.2 \mathrm{~Hz}, 1 \mathrm{H}), 7.51-7.45(\mathrm{~m}, 2 \mathrm{H}), 3.92-3.85(\mathrm{~m}, 5 \mathrm{H}), 3.53(\mathrm{~s}, 2 \mathrm{H})$, 3.80-3.32 (m, 2H), 2.81-2.77 (m, 4H), 1.73-1.69 (m, 2H), 1.56- $1.46(\mathrm{~m}, 2 \mathrm{H}) ;{ }^{13} \mathrm{C}$ NMR $\left(100 \mathrm{MHz}\right.$, DMSO- $\left.d_{6}\right): \delta 160.62,140.6$, 138.7, 136.7, 135.5, 134.3 (2C), 132.3, 131.5, 128.3, 115.8, 112.8, 65.09 (2C), 59.12, 56.21, 55.32, 55.16, 36.44 (2C), 22.2; MS: calcd. for $\mathrm{C}_{21} \mathrm{H}_{23} \mathrm{~N}_{3} \mathrm{O}_{2} \mathrm{~S}$ [m/z] 381.49; found: 382.53 $[\mathrm{M}+\mathrm{H}]^{+}$.

5-(2,4,6-Triethylbenzyl)-4,5,6,7-tetrahydro- $\mathrm{N}$-(tetrahydro-2H-pyran-4-yl)thieno[3,2-c]pyridine-2-carboxamide (7F): Off white solid; Yield: $155 \mathrm{mg}$, 85 \%; m.p.: 163$165{ }^{\circ} \mathrm{C}$; Elemental analysis, $\mathrm{C}_{26} \mathrm{H}_{36} \mathrm{~N}_{2} \mathrm{O}_{2} \mathrm{~S}$, calcd. (found) $\%$ : C 70.87 (70.89), H 8.23 (8.25), N 6.36 (6.38). IR ( $\left.\mathrm{KBr}, \mathrm{v}_{\max }, \mathrm{cm}^{-1}\right)$ : 3307, 2962, 2837, 2763, 1618, 1568, 1532, 1459, 1367, 1323, 1240, 1139, 1093, 1008, 872, 618; 'H NMR (400 MHz, DMSO- $\left.\left.d_{6}\right)\right) \delta: 8.10(\mathrm{~d}, J=8.0 \mathrm{~Hz}, 1 \mathrm{H}), 7.45(\mathrm{~s}, 1 \mathrm{H}), 6.87(\mathrm{~s}$, $2 \mathrm{H}), 3.85(\mathrm{~d}, J=11.6 \mathrm{~Hz}, 3 \mathrm{H}), 3.64(\mathrm{~s}, 1 \mathrm{H}), 3.38-3.28(\mathrm{~m}$, $2 \mathrm{H}), 2.72-2.68(\mathrm{~m}, 8 \mathrm{H}), 2.56-2.50(\mathrm{~m}, 3 \mathrm{H}), 1.69$ (brs, $2 \mathrm{H})$, 1.54-1.48 (m, 2H), 1.23-1.10 (m, 8H); ${ }^{13} \mathrm{C}$ NMR (100 MHz, DMSO- $\left.d_{6}\right): \delta 161.92,146.6,142.7,136.7,136.2(2 \mathrm{C}), 135.5$, 134.3 (2C), 131.5, 65.11 (2C), 57.12, 56.6, 55.7, 55.16, 36.34 (2C), 27.8, 26.2 (2C), 22.2, 14.6 (2C), 14.3; MS: calcd. for $\mathrm{C}_{26} \mathrm{H}_{36} \mathrm{~N}_{2} \mathrm{O}_{2} \mathrm{~S}[\mathrm{~m} / \mathrm{z}]$ 440.64; found: $441.33[\mathrm{M}+\mathrm{H}]^{+}$.

4,5,6,7-Tetrahydro-N-(tetrahydro-2H-pyran-4-yl)-5[(1-methyl-1H-pyrazol-4-yl)methyl]thieno[3,2-c]pyridine2-carboxamide (7G): White solid; Yield: $124 \mathrm{mg}, 83 \%$; m.p.: 164-168 ${ }^{\circ} \mathrm{C}$; Elemental analysis, $\mathrm{C}_{18} \mathrm{H}_{24} \mathrm{~N}_{4} \mathrm{O}_{2} \mathrm{~S}$, calcd. (found) \%: C 59.97 (59.95), H 6.71 (6.73), N 15.54 (15.52). IR (KBr, $v_{\max }$, $\left.\mathrm{cm}^{-1}\right)$ : 3494, 3274, 2930, 2841, 1618, 1540, 1461, 1324, 1139, 1087, 984, 871, 792, 677; ${ }^{1} \mathrm{H}$ NMR (400 MHz, DMSO-d $\left.d_{6}\right) \delta$ : $8.45(\mathrm{~s}, 1 \mathrm{H}), 8.25(\mathrm{~s}, 1 \mathrm{H}), 8.13(\mathrm{~d}, J=8.0 \mathrm{~Hz}, 1 \mathrm{H}), 7.97(\mathrm{~s}, 1 \mathrm{H})$, $7.72(\mathrm{~d}, J=8.0 \mathrm{~Hz}, 1 \mathrm{H}), 7.61(\mathrm{~d}, J=7.6 \mathrm{~Hz}, 1 \mathrm{H}), 7.44(\mathrm{~s}, 1 \mathrm{H})$, 3.90 (s, 2H), 3.88-3.84 (m, 5H), 3.68 (s, 2H), 3.48 (s, 2H), 2.80 $(\mathrm{s}, 2 \mathrm{H}), 2.73(\mathrm{~d}, J=5.2 \mathrm{~Hz}, 2 \mathrm{H}), 1.71(\mathrm{~d}, J=1.6 \mathrm{~Hz}, 2 \mathrm{H}), 1.53-$ $1.49(\mathrm{~m}, 2 \mathrm{H}) ;) ;{ }^{13} \mathrm{C}$ NMR $\left(100 \mathrm{MHz}\right.$, DMSO- $\left.d_{6}\right): \delta 161.1,139.5$, 137.4 (2C), 136.9, 135.7, 125.2, 116.0, 65.6 (2C), 56.9, 55.7, 55.2 (2C), 40.5, 36.7 (2C), MS: calcd. for $\mathrm{C}_{18} \mathrm{H}_{24} \mathrm{~N}_{4} \mathrm{O}_{2} \mathrm{~S}[\mathrm{~m} / \mathrm{z}]$ 360.47; found: $361.43[\mathrm{M}+\mathrm{H}]^{+}$.

4,5,6,7-Tetrahydro-N-(tetrahydro-2H-pyran-4-yl)-5[(6-(1-methyl-1H-pyrazol-4-yl)pyridin-3-yl)methyl]thieno[3,2-c]pyridine-2-carboxamide $\mathbf{( 7 H )}$ : Light yellow solid; Yield: $140 \mathrm{mg}, 77 \%$; m.p.: $171-174{ }^{\circ} \mathrm{C}$; Elemental analysis, $\mathrm{C}_{23} \mathrm{H}_{27} \mathrm{~N}_{5} \mathrm{O}_{2} \mathrm{~S}$, calcd. (found) \%: C 63.13 (63.11), H 6.22 (6.20), $\mathrm{N} 16.01$ (15.99). IR (KBr, $\left.v_{\max }, \mathrm{cm}^{-1}\right): 3297,2944,2356,1613$, 1531, 1454, 1319, 1290, 1137, 1089, 968, 863; ${ }^{1} \mathrm{H}$ NMR (400 MHz, DMSO- $\left.d_{6}\right) \delta: 8.11(\mathrm{~d}, J=8.0 \mathrm{~Hz}, 1 \mathrm{H}), 7.60(\mathrm{~s}, 1 \mathrm{H}), 7.43$ $(\mathrm{s}, 1 \mathrm{H}), 7.33(\mathrm{~s}, 1 \mathrm{H}), 3.98-3.84(\mathrm{~m}, 3 \mathrm{H}), 3.82(\mathrm{~s}, 3 \mathrm{H}), 3.54(\mathrm{~s}$, $2 \mathrm{H}), 3.42-3.32(\mathrm{~m}, 5 \mathrm{H}), 2.80(\mathrm{~s}, 2 \mathrm{H}), 2.73(\mathrm{~s}, 2 \mathrm{H}), 1.65-1.62$ (m, 2H), 1.51-1.40 (m, 2H); ${ }^{13} \mathrm{C}$ NMR (100 MHz, DMSO- $\left.d_{6}\right)$ : $\delta 160.45,150.67,149.64,138.21,137.26,136.96,134.48,130.78$, $129.35,126.28,122.68,118.89,66.10$ (2C), 57.86, 51.96, 49.33, 45.63, 38.70, 32.48 (2C), 25.09. MS: calcd. for $\mathrm{C}_{23} \mathrm{H}_{27} \mathrm{~N}_{5} \mathrm{O}_{2} \mathrm{~S}$ [m/z] 437.56; found: $438.4[\mathrm{M}+\mathrm{H}]^{+}$.

5-(Cyclobutylmethyl)-4,5,6,7-tetrahydro-N-(tetrahydro-2H-pyran-4-yl)thieno[3,2-c]pyridine-2-carboxamide (7I): Off white solid; Yield: $103 \mathrm{mg}, 74$ \%; m.p.: 151$153{ }^{\circ} \mathrm{C}$; Elemental analysis, $\mathrm{C}_{18} \mathrm{H}_{26} \mathrm{~N}_{2} \mathrm{O}_{2} \mathrm{~S}$, calcd. (found) \%: C 64.64 (64.65), H 7.84 (7.86), N 8.38 (8.37). IR (KBr, $v_{\max }$, $\left.\mathrm{cm}^{-1}\right): 3531,3275,2955,2929,2759,1705,1615,1537,1459$, 1434, 1368, 1326, 1238, 1131, 1086, 1009, 977, 873, 793, 660, 539, 508; ${ }^{1} \mathrm{H}$ NMR (400 MHz, DMSO- $\left.d_{6}\right) \delta: 8.19(\mathrm{~d}, J=7.6$ 
$\mathrm{Hz}, 1 \mathrm{H}), 7.46$ (s, 1H), 3.91-3.84 (m, 3H), 3.39-3.34 (m, 4H), 3.29-3.23 (m, 2H), 2.77-2.67 (m, 4H), 2.06-1.99 (m, 2H), 1.911.65 (m, 6H), 1.58-1.51 (m, 2H), 1.30 (brs, $1 \mathrm{H}) ;{ }^{13} \mathrm{C}$ NMR $\left(100 \mathrm{MHz}, \mathrm{DMSO}-d_{6}\right): \delta 160.17,140.55,136.44,134.79$, $126.31,65.10$ (2C), 61.34, 50.96, 49.61, 45.31, 34.47 (2C), 29.22, 24.8 (2C), 22.51, 18.74; MS: calcd. for $\mathrm{C}_{18} \mathrm{H}_{26} \mathrm{~N}_{2} \mathrm{O}_{2} \mathrm{~S}$ $[\mathrm{m} / \mathrm{z}]$ 334.48; found: $335.21[\mathrm{M}+\mathrm{H}]^{+}$.

5-(Cyclopropylmethyl)-4,5,6,7-tetrahydro- $\mathrm{N}$-(tetrahydro-2H-pyran-4-yl)thieno[3,2-c]pyridine-2-carboxamide (7J): Off white solid; Yield: $101 \mathrm{mg}, 76 \%$; m.p.: 154$156{ }^{\circ} \mathrm{C}$; Elemental analysis, $\mathrm{C}_{17} \mathrm{H}_{24} \mathrm{~N}_{2} \mathrm{O}_{2} \mathrm{~S}$, calcd. (found) \%: C 63.72 (63.70), H 7.55 (7.53), N 8.74 (8.75). IR (KBr, $v_{\max }$, $\left.\mathrm{cm}^{-1}\right): 3289,2923,2842,1619,1539,1462,1322,1239,1137$, $1085,873,660 ;{ }^{1} \mathrm{H}$ NMR $\left(400 \mathrm{MHz}\right.$, DMSO- $\left.d_{6}\right) \delta: 8.15(\mathrm{~d}, J$ $=6.4 \mathrm{~Hz}, 1 \mathrm{H}), 7.48(\mathrm{~s}, 1 \mathrm{H}), 3.91-3.68(\mathrm{~m}, 3 \mathrm{H}), 3.51(\mathrm{~s}, 2 \mathrm{H})$, $3.38-3.33(\mathrm{~m}, 2 \mathrm{H}), 2.80-2.76(\mathrm{~m}, 4 \mathrm{H}), 2.37(\mathrm{~d}, J=5.2 \mathrm{~Hz}$, $2 \mathrm{H}), 1.72(\mathrm{~d}, J=10.0 \mathrm{~Hz}, 2 \mathrm{H}), 1.56-1.50(\mathrm{~m}, 2 \mathrm{H}), 0.89$ (d, $J$ $=4.4 \mathrm{~Hz}, 1 \mathrm{H}), 0.49(\mathrm{~d}, 6.0 \mathrm{~Hz}, 2 \mathrm{H}), 0.12(\mathrm{~d}, J=3.2 \mathrm{~Hz}, 2 \mathrm{H})$; ${ }^{13} \mathrm{C}$ NMR (100 MHz, DMSO- $\left.d_{6}\right): \delta 160.47,138.23,136.74$, 134.79, 126.31, 66.10 (2C), 61.84, 51.96, 49.81, 45.61, 32.47 (2C), 25.22, 8.51, 3.71 (2C); MS: calcd. for $\mathrm{C}_{17} \mathrm{H}_{24} \mathrm{~N}_{2} \mathrm{O}_{2} \mathrm{~S}[\mathrm{~m} / \mathrm{z}]$ 320.45; found: $321.5[\mathrm{M}+\mathrm{H}]^{+}$.

4,5,6,7-Tetrahydro-N-(tetrahydro-2H-pyran-4-yl)-5(2-(naphthalen-2-yl)-2-oxoethyl)thieno[3,2-c]pyridine-2carboxamide (7K): Light yellow solid; Yield: $143 \mathrm{mg}, 80 \%$; m.p.: $160-163{ }^{\circ} \mathrm{C}$; Elemental analysis, $\mathrm{C}_{25} \mathrm{H}_{26} \mathrm{~N}_{2} \mathrm{O}_{3} \mathrm{~S}$, calcd. (found) \%: C 69.10 (69.13), H 6.03 (6.06), N 6.45 (6.46). IR $\left(\mathrm{KBr}, v_{\max }, \mathrm{cm}^{-1}\right): 3248,3056,2953,2914,2841,1687,1608$, 1530, 1457, 1375, 1332, 1293, 1242, 1165, 1123, 1091, 1004, $869,804,752,675,579 ;{ }^{1} \mathrm{H}$ NMR $\left(400 \mathrm{MHz}, \mathrm{DMSO}-d_{6}\right) \delta$ : $8.72(\mathrm{~s}, 1 \mathrm{H}), 8.17-8.11(\mathrm{~m}, 2 \mathrm{H}), 8.02-7.96(\mathrm{~m}, 2 \mathrm{H}), 7.99(\mathrm{~s}, 1 \mathrm{H})$, 7.69-7.60 (m, 2H), $7.46(\mathrm{~s}, 1 \mathrm{H}), 4.25(\mathrm{~s}, 2 \mathrm{H}), 3.92-3.84(\mathrm{~m}, 3 \mathrm{H})$, 3.69 (s, 2H), 3.38-3.32 (m, 2H), 2.94 (brs, $2 \mathrm{H}), 2.83$ (brs, $2 \mathrm{H}$ ), $1.72(\mathrm{~d}, J=12.4 \mathrm{~Hz}, 2 \mathrm{H}), 1.56-1.48(\mathrm{~m}, 2 \mathrm{H}),{ }^{13} \mathrm{C}$ NMR $(100$ $\left.\mathrm{MHz}, \mathrm{DMSO}-d_{6}\right): \delta 197.11,160.43,138.02,136.84,135.02$, $134.40,133.09,132.07,129.76,129.53,128.58,128.14,127.61$, 126.87, 126.18, 123.68, 66.08 (2C), 62.59, 51.69, 49.76, 45.61, 32.45 (2C), 25.10; MS: calcd. for $\mathrm{C}_{25} \mathrm{H}_{26} \mathrm{~N}_{2} \mathrm{O}_{3} \mathrm{~S}[\mathrm{~m} / \mathrm{z}] 434.55$; found: $435.53[\mathrm{M}+\mathrm{H}]^{+}$

5-[2-(3,4-Difluorophenyl)-2-oxoethyl]-N-(tetrahydro2H-pyran-4-yl)-4,5,6,7-tetrahydrothieno[3,2-c]pyridine-2carboxamide (7L): Light Brown solid; Yield: $145 \mathrm{mg}, 83 \%$; m.p.: $158-162{ }^{\circ} \mathrm{C}$; Elemental analysis, $\mathrm{C}_{21} \mathrm{H}_{22} \mathrm{~N}_{2} \mathrm{O}_{3} \mathrm{SF}_{2}$, calcd. (found) \%: C 59.99 (59.94), H 5.27 (5.28), N 6.66 (6.64). IR $\left(\mathrm{KBr}, v_{\max }, \mathrm{cm}^{-1}\right): 3248,2955,2914,2841,1695,1608,1530$, 1467, 1332, 1293, 1175, 1123, 1091, 1004, 869, 806, 754, 675, $670 ;{ }^{1} \mathrm{H}$ NMR $\left(400 \mathrm{MHz}\right.$, DMSO- $\left.d_{6}\right) \delta: 8.16(\mathrm{~d}, J=6.0 \mathrm{~Hz}, 1 \mathrm{H})$, 8.06-8.02 (m, 2H), $7.91(\mathrm{~s}, 1 \mathrm{H}), 7.44(\mathrm{~s}, 1 \mathrm{H}), 4.06(\mathrm{~s}, 2 \mathrm{H}) ;{ }^{13} \mathrm{C}$ NMR (100 MHz, DMSO- $\left.d_{6}\right)$ : $\delta 194.6,160.78,152.75,149.1$, $141.73,137.75,136.92,135.83,132.96,126.08,125.44,115.34$, 71.26, 66.06 (2C), 54.57, 52.88, 51.60, 32.44 (2C), 22.43; MS: calcd. for $\mathrm{C}_{21} \mathrm{H}_{22} \mathrm{~N}_{2} \mathrm{O}_{3} \mathrm{SF}_{2}[\mathrm{~m} / \mathrm{z}] 420.47$; found: $421.53[\mathrm{M}+\mathrm{H}]^{+}$.

5-(2-Cyclopropyl-2-oxo-1-phenylethyl)-4,5,6,7tetrahydro-N-(tetrahydro-2H-pyran-4-yl)thieno[3,2c]pyridine-2-carboxamide (7M): Semi solid; Yield: $155 \mathrm{mg}$, $85 \%$; Elemental analysis, $\mathrm{C}_{24} \mathrm{H}_{28} \mathrm{~N}_{2} \mathrm{O}_{3} \mathrm{~S}$, calcd. (found) \%: $\mathrm{C}$ 67.90 (67.87), H 6.65 (66.67), N 6.60 (6.56). IR (KBr, $v_{\max }$, $\left.\mathrm{cm}^{-1}\right)$ : 3322, 2925, 2849, 1702, 1626, 1572, 1532, 1485, 1456,
$1379,1238,1088,1009,757,575 ;{ }^{~} \mathrm{H}$ NMR $(400 \mathrm{MHz}$, DMSO$\left.d_{6}\right) \delta: 8.10(\mathrm{~d}, J=6.0 \mathrm{~Hz}, 1 \mathrm{H}), 7.52(\mathrm{t}, J=5.6 \mathrm{~Hz}, 1 \mathrm{H}), 7.43-$ $7.41(\mathrm{~m}, 2 \mathrm{H}), 7.28-7.25(\mathrm{~m}, 2 \mathrm{H}), 4.81(\mathrm{~s}, 1 \mathrm{H}), 3.90-3.84(\mathrm{~m}, 3 \mathrm{H})$, 3.54 (q, $J=5.2 \mathrm{~Hz}, 2 \mathrm{H}), 3.37-3.32(\mathrm{~m}, 1 \mathrm{H}), 2.83$ (brs, 3H), $2.71(\mathrm{t}, J=7.2 \mathrm{~Hz}, 1 \mathrm{H}), 2.38(\mathrm{~d}, J=3.6 \mathrm{~Hz}, 1 \mathrm{H}), 1.71(\mathrm{~d}, J=$ $9.6 \mathrm{~Hz}, 2 \mathrm{H}), 1.54-1.47$ (m, 2H), 1.23 (s, 1H), 0.90-0.89 (m, 5H); ${ }^{13} \mathrm{C}$ NMR (100 MHz, DMSO- $\left.d_{6}\right): \delta 207.39,170.31,161.58$, $160.38,138.05,136.98,134.13,130.72,130.17,126.27,124.70$, $115.83,115.65,71.26,66.06$ (2C), 49.67, 47.56, 45.60, 32.44 (2C), 25.29, 17.71, 11.58, 11.19; MS: calcd. for $\mathrm{C}_{24} \mathrm{H}_{28} \mathrm{~N}_{2} \mathrm{O}_{3} \mathrm{~S}$ $[\mathrm{m} / \mathrm{z}]$ 442.5; found: $443.6[\mathrm{M}+\mathrm{H}]^{+}$.

Anticancer activity: Human lung carcinoma (HCT-116) cell lines were obtained from NCCS Pune and were kept in RPMI 1640 medium (\#AL199A, Himedia) supplemented with $10 \%$ Fetal Bovine Serum (\#RM10432, Himedia). The cells were made to confluency at $37{ }^{\circ} \mathrm{C}$ in a $\mathrm{CO}_{2}$ incubator (Healforce, China) with humidified atmosphere and $5 \% \mathrm{CO}_{2}$. Seeded 200 $\mu \mathrm{L}$ cell suspension in a 96-well plate at required cell density (20,000 cells per well) without the test agent and allowed the cells to grow for about $12 \mathrm{~h}$. Test samples in the concentrations of $25 \mu \mathrm{g}, 50 \mu \mathrm{g}, 75 \mu \mathrm{g}, 100 \mu \mathrm{g}, 125 \mu \mathrm{g} / \mathrm{mL}$ of compounds and $50 \mu \mathrm{g} / \mathrm{mL}$ drugs were added for the growth of the cells separately and incubated the plate for $24 \mathrm{~h}$ at $37^{\circ} \mathrm{C}$ in a $5 \% \mathrm{CO}_{2}$ atmosphere. After the incubation period, the plates were taken out of the incubator and removed spent media and added MTT reagent (camptothecin) to a final concentration of $0.5 \mathrm{mg} / \mathrm{mL}$ of total volume and the plates were incubated for $3 \mathrm{~h}$, removed the MTT reagent and $100 \mu \mathrm{L}$ of solubilization solution (DMSO) was added. The absorbance was recorded on an ELISA reader at $570 \mathrm{~nm}$ and $630 \mathrm{~nm}$ used as the reference wavelength. The $\mathrm{IC}_{50}$ value was obtained by using linear regre-ssion equation i.e., $\mathrm{y}=\mathrm{mx}+\mathrm{c}$ where, $\mathrm{y}=50, \mathrm{~m}$ and $\mathrm{c}$ values were derived from the viability graph.

\section{RESULTS AND DISCUSSION}

The synthesis of 5-substituted 4,5,6,7-tetra-hydro$\mathrm{N}$-(tetrahydro-2H-pyran-4-yl)thieno[3,2-c]pyridine-2-carboxamide derivatives (7A-M) is illustrated in Scheme-I. Formylation of tert-butyl 4-oxopiperidine-1-carboxylate (1) under Villsmeyer-Hack protocol was carried out in presence of DMF and $\mathrm{POCl}_{3}$ to achieve the desired compound tert-butyl 3-formyl-4-oxopiperidine-1-carboxylate (2). Treatment of aldehyde 2 with 2-mercaptoacetate in presence of triethyl amine in dichloromethane at $45{ }^{\circ} \mathrm{C}$ for $16 \mathrm{~h}$ produced the cyclized product, 5-tert-butyl-2-ethyl-6,7-dihydrothieno[3,2c]pyridine-2,5(4H)-dicarboxylate (3). Alkaline hydrolysis of ethyl ester $\mathbf{3}$ followed by coupling with tetrahydro- $2 \mathrm{H}$-pyran4-amine in presence of EDC.HCl, HOBt and di-isopropyl amine in DMF at room temperature for $5 \mathrm{~h}$ produced the desired compound N-(tetrahydro-2H-pyran-4-yl)-4,5,6,7-tetrahydrothieno[3,2-c]pyridine-2-carboxamide (6). De-protection of the Boc group in presence of trifluoroacetic acid followed by coupling with various aryl/alkyl/acyl halides in presence of $\mathrm{K}_{2} \mathrm{CO}_{3}$ in DMF at room temperature for $16 \mathrm{~h}$ resulted in the formation of desired compounds 7A-M in moderate yields.

These derivatives have been characterized by ${ }^{1} \mathrm{H}$ NMR, mass and IR spectroscopic tools. The structural determination of 5-(2-chlorobenzyl)-4,5,6,7-tetrahydro- $\mathrm{N}$-(tetrahydro- $2 \mathrm{H}$ - 

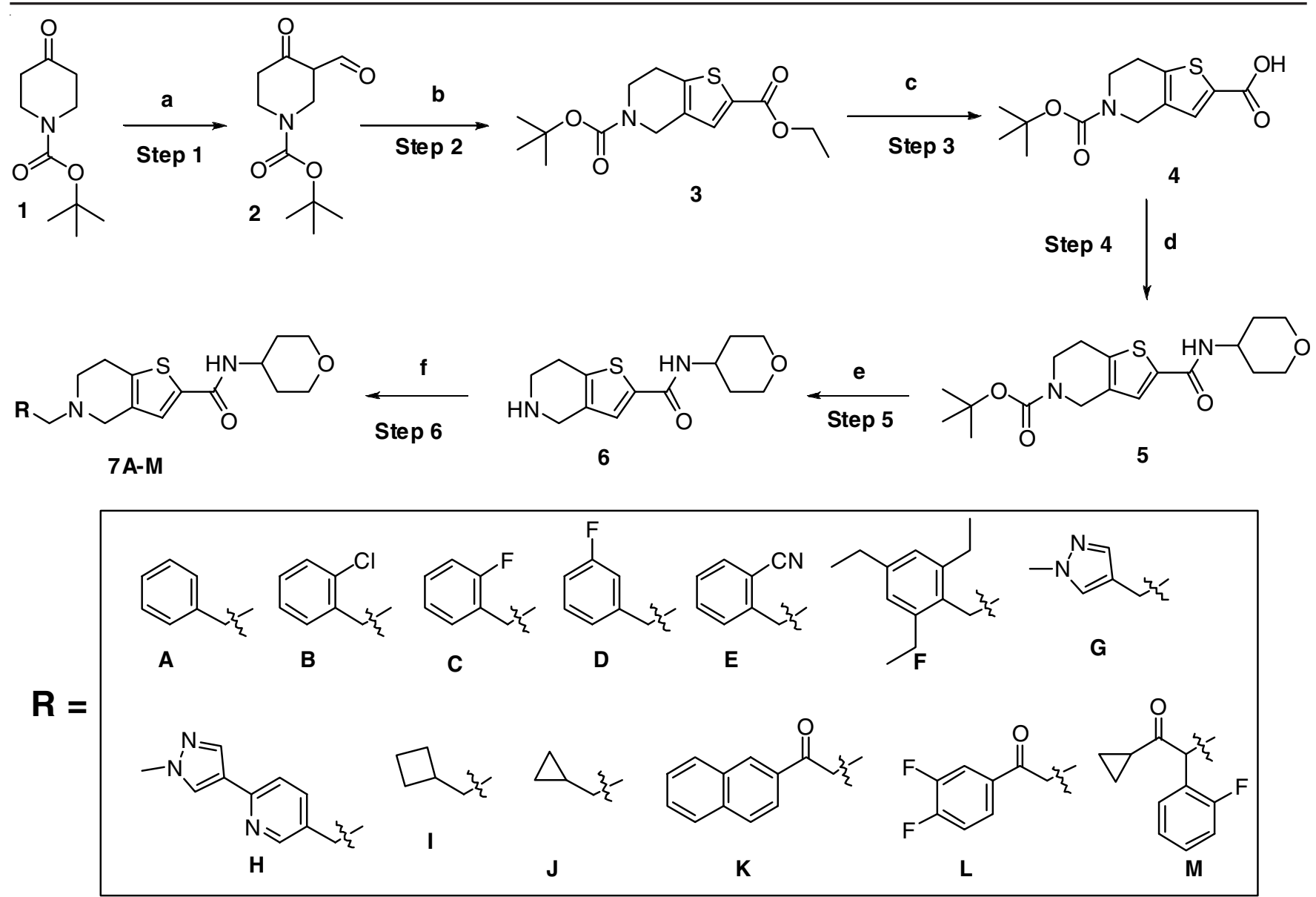

Reaction conditions: a) DMF, $\mathrm{POCl}_{3}, \mathrm{DCM}, 0{ }^{\circ} \mathrm{C}$-room temperature, $4 \mathrm{~h}$; b) 2-mercaptoacetate, triethyl amine, $\mathrm{DCM}, 45^{\circ} \mathrm{C}$, $16 \mathrm{~h}$; c) $\mathrm{NaOH}$, THF:MeOH: $\mathrm{H}_{2} \mathrm{O}$, room temperature, $16 \mathrm{~h}$; d) tetrahydro- $2 \mathrm{H}$-pyran-4-amine, EDC.HCl, HOBt, di-isopropyl amine, DMF, room temperature, $5 \mathrm{~h}$; e) TFA, DCM, $0{ }^{\circ} \mathrm{C}$-room temperature, $16 \mathrm{~h}$; f) $\mathrm{R}-\mathrm{X}, \mathrm{K}_{2} \mathrm{CO}_{3}$, DMF, room temperature, $16 \mathrm{~h}$

Scheme-I: Synthesis of novel 5-substituted 4,5,6,7-tetrahydro-N-(tetrahydro-2H-pyran-4-yl)thieno[3,2-c]pyridine-2-carboxamide derivatives (7A-7M)

pyran-4-yl)thieno[3,2-c]pyridine-2-carboxamide (7B). In ${ }^{1} \mathrm{H}$ NMR, the proton signals resonating at $8.15 \mathrm{ppm}$ (doublet) and $7.36 \mathrm{ppm}$ (singlet) corresponds to $-\mathrm{NH}$ and thiophene ring proton, while the proton signals resonating at 7.53, 7.45, 7.33 and $7.23 \mathrm{ppm}$ as doublets is assigned to 2-chloro-phenyl ring, respectively. The characteristic benzylic $-\mathrm{CH}_{2}$ - resonated at 3.77 ppm as singlet and the remaining aliphatic protons such as piperidine and morpholine ring protons resonated in the expected region. The structure of compound $\mathbf{7 B}$ was further confirming by its molecular weight $\mathrm{m} / \mathrm{z}, 390.76$ (LC-MS purity: $93.86 \%$ ) which was found to be in agreement with the desired structure. In the IR spectra of compound $\mathbf{7 B}$, the characteristic peaks in the region $3280,3065,2949,1614,1571 \mathrm{~cm}^{-1}$ are assigned to $-\mathrm{NH}, \mathrm{Ar}-\mathrm{C}-\mathrm{H}$, aliph. $-\mathrm{C}-\mathrm{H}$, $-\mathrm{CO}-\mathrm{NH}-$ and $-\mathrm{C}=\mathrm{C}$ - groups respectively. The above spectroscopic techniques, thus confirms the structure of compound 7B. Similarly, the structures of remaining compounds in the series have been confirmed.

Anticancer activity: Compounds (7A-M) were screened for their in vitro cytotoxic potential against human lung carcinoma (HCT-116) cell line using the MTT assay and the obtained $\mathrm{IC}_{50}$ values are tabulated in Table-1. Among all the compound 7B $\left(\mathrm{IC}_{50}: 69.52 \mu \mathrm{g}\right)$ and compound $7 \mathrm{~K}\left(\mathrm{IC}_{50}: 66.35 \mu \mathrm{g}\right)$ showed significant potent activity at micro molar concentration when compared with the standard drug camptothecin, while the compounds 7C, 7D, 7E, 7G, 7H and 7M exhibited moderate activity and compounds $\mathbf{7 A}, \mathbf{7 F}, \mathbf{7 I}, \mathbf{7} \mathbf{J}$ and $\mathbf{7 L}$ showed weak activity (Table-1). It is evident from the cytotoxic activity results that the compounds containing 2-chloro-benzyl (7B) and 2-acetyl naphthalene (7K) ring substituted to the main scaffold exhibited potent anticancer activities compared to the standard drug.

TABLE-1 in vitro CYTOTOXIC ACTIVITY OF COMPOUNDS (7A-M)

\begin{tabular}{cc|cc}
\hline Compound & $\mathrm{IC}_{50}(\mu \mathrm{g} / \mathrm{mL})$ & Compound & $\mathrm{IC}_{50}(\mu \mathrm{g} / \mathrm{mL})$ \\
\hline 7A & $82.36 \pm 0.02$ & $\mathbf{7 H}$ & $72.15 \pm 0.01$ \\
7B & $69.52 \pm 0.02$ & $\mathbf{7 I}$ & $80.35 \pm 0.01$ \\
$\mathbf{7 C}$ & $78.48 \pm 0.01$ & $\mathbf{7 J}$ & $80.21 \pm 0.02$ \\
7D & $77.21 \pm 0.02$ & $\mathbf{7 K}$ & $66.35 \pm 0.02$ \\
$\mathbf{7 E}$ & $75.35 \pm 0.01$ & $\mathbf{7 L}$ & $83.25 \pm 0.01$ \\
$\mathbf{7 F}$ & $80.05 \pm 0.01$ & $\mathbf{7 M}$ & $71.25 \pm 0.01$ \\
$\mathbf{7 G}$ & $79.45 \pm 0.01$ & Camptothecin $(\mathrm{SD})$ & $47.36 \pm 0.02$ \\
\hline
\end{tabular}

\section{Conclusion}

In conclusion, we have described the synthesis and characterization of some novel 5-substituted 4,5,6,7-tetrahydro-N(tetrahydro-2H-pyran-4-yl)thieno[3,2-c]pyridine-2-carboxamide derivatives (7A-M). These derivatives were screened for their in vitro cytotoxic potential against human lung carci- 
noma (HCT-116) cell line using the MTT assay. The anticancer activity results revealed that compound $\mathbf{7 B}\left(\mathrm{IC}_{50}: 69.52 \mu \mathrm{g}\right)$ and compound $7 \mathrm{~K}\left(\mathrm{IC}_{50}: 66.35 \mu \mathrm{g}\right)$ showed moderate potent activity at micro molar concentration when compared with the standard drug camptothecin, while compounds 7C, 7D, 7E, 7G, 7H and 7M exhibited moderate activity and compounds 7A, 7F, 7I, 7J and 7L showed weak activity.

\section{CONFLICT OF INTEREST}

The authors declare that there is no conflict of interests regarding the publication of this article.

\section{REFERENCES}

1. J.N. Sangshetti, P.P. Dharmadhikari, R.S. Chouthe, B. Fatema, V. Lad, V. Karande, S.N. Darandale and D.B. Shinde, Bioorg. Med. Chem. Lett., 23, 2250 (2013);

https://doi.org/10.1016/j.bmcl.2013.01.041.

2. R. Kalaria, M. Mittal, C. Rajyaguru and J.J. Upadhyay, J. Chem. Pharm. Res., 4, 1566 (2012).

3. R. Kalaria, R.J. Odedara, R.S. Dave, C. Rajyaguru and J.J. Upadhyay, J. Appl. Technol. Environ. Sanit., 2, 109 (2012).

4. J. Kedia, K.S. Nimavant and K.B. Vyas, J. Chem. Pharm. Res., 4, 1864 (2012).

5. Y.S. Patel, P.N. Patel and H.S. Patel, Int. Res. J. Pure Appl. Chem., 4, 315 (2014); https://doi.org/10.9734/IRJPAC/2014/5955.

6. V.P. Modi, P.N. Patel and H.S. Patel, Der Pharm. Lett., 3, 120 (2011).

7. J.N. Sangshetti, A.S. Zambare, F.A. Khan, I. Gonjari and Z. Zaheer, Mini Rev. Med. Chem., 14, 988 (2014); https://doi.org/10.2174/1389557514666141106131425.

8. M. Mittal, S.M. Sarode and G. Vidyasagar, Int. J. Pharm. Biosci., 2, 188 (2011)

9. D.P. Kawade and P.B. Khedekar, Der. Pharm. Chem., 4, 1856 (2012).

10. P.N. Patel, D.J. Patel and H.S. Patel, Appl. Organomet. Chem., 25, 454 (2011); https://doi.org/10.1002/aoc.1786.

11. H.M. Parekh, P.B. Pansuriya and M.N. Patel, Pol. J. Chem., 79, 1843 (2005).

12. B.G. Tweedy, Phytopathology, 55, 910 (1964).

13. B.B. Lohray, V.B. Lohray, B.K. Srivastava, P.B. Kapadnis and P. Pandya, Bioorg. Med. Chem., 12, 4557 (2004); https://doi.org/10.1016/j.bmc.2004.07.019.

14. S.N. Darandale, N.A. Mulla, D.N. Pansare, J.N. Sangshetti and D.B. Shinde, Eur. J. Med. Chem., 65, 527 (2013); https://doi.org/10.1016/j.ejmech.2013.04.045.

15. J.N. Sangshetti, F.A. Kalam Khan, R.S. Chouthe, M.G. Damale and D.B. Shinde, Chin. Chem. Lett., 25, 1033 (2014); https://doi.org/10.1016/j.cclet.2014.04.003.

16. J.N. Sangshetti, R.I. Shaikh, F.A.K. Khan, H. Patil, S.D. Marathe, W.N. Gade and D.B. Shinde, Bioorg. Med. Chem. Lett., 24, 1605 (2014); https://doi.org/10.1016/j.bmcl.2014.01.035.
17. A.M.R. El-Sayed, A.G. Abdel-Hafez, N.N.S. Mohamed and S.F. Abdalla, Turk. J. Chem., 33, 421 (2009).

18. J.P. Maffrand and J.P. Frehel, Antiinflammatory Thieno[2,3-c]pyridine Derivatives, US Patent 4,496,568 (1985)

19. A. Amselen, Thieno-pyridine Derivatives, Process for their Preparation and their Applications, US Patent 3,983,125 (1976).

20. A. Amselem, 5-O-Cyanobenzyl-4,5,6,7-tetrahydrothieno[3,2-c]-pyridine Maleate, US Patent 4,097,482 (1978).

21. A.R.J. Castaigne, Thieno[3,2-c]pyridine Derivatives, US Patent 4,051,141 (1977).

22. J.P. Maffrand, Thieno[2,3-C]pyridine Derivatives and Therapeutic Composition Containing same, US Patent 4,075,340 (1978).

23. M. Fujita, T. Seki, H. Inada and N. Ikeda, Bioorg. Med. Chem. Lett., 12, 1607 (2002) https://doi.org/10.1016/S0960-894X(02)00228-7.

24. C.S. Schneider, K.H. Weber, H. Daniel, W.D. Bechtel and K. BoekeKuhn, J. Med. Chem., 27, 1150 (1984); https://doi.org/10.1021/jm00375a011.

25. L. Zheng, J. Xiang and X.A. Bai, J. Heterocycl. Chem., 43, 321 (2006); https://doi.org/10.1002/ihet.5570430211.

26. R. Romagnoli, P.G. Baraldi, M.D. Carrion, O. Cruz-Lopez, C.L. Cara, M. Tolomeo, S. Grimaudo, A.D. Cristina, M.R. Pipitone, J. Balzarini, S. Kandil, A. Brancale, T. Sarkar and E. Hamel, Bioorg. Med. Chem. Lett., 18, 5041 (2008); https://doi.org/10.1016/j.bmcl.2008.08.006

27. R. Busacca, Compounds having Antiplatelet Aggregation Activity and Pharmaceutical Compositions Containing them, US Patent 4,737,502 (1988).

28. H. Koike, F. Asai, A. Sugidachi, T. Kimura, T. Inoue, S. Nishino and Y. Tsuzaki, Tetrahydrothienopyridine Derivatives, Furo and Pyrrolo Analogs Thereof for Inhibiting Blood Platelet Aggregation, US Patent 5,436,242 (1995)

29. P. Madsen, J.M. Lundbeck, P. Jakobsen, A.R. Varming and N. Westergaard, Bioorg. Med. Chem., 8, 2277 (2000); https://doi.org/10.1016/S0968-0896(00)00153-X.

30. P. Madsen, J.M. Lundbeck, N. Westergaard and P. Jakobsen, 4,5,6,7Tetrahydro-thieno(2,3-C)pyridine Derivatives, US Patent 6,090,797 (2000).

31. G. Samala, P.B. Devi, R. Nallangi, J.P. Sridevi, S. Saxena, P. Yogeeswari and D. Sriram, Bioorg. Med. Chem. Lett., 22, 1938 (2014); https://doi.org/10.1016/j.bmc.2014.01.030.

32. R. Nallangi, G. Samala, G.P. Sridevi, P. Yogeeswari and D. Sriram, Eur. J. Med. Chem., 76, 110 (2014); https://doi.org/10.1016/j.ejmech.2014.02.028.

33. B.K. Srivastava, M. Solanki, B. Mishra, R. Soni, S. Jayadev, D. Valani, M. Jain and P.R. Patel, Bioorg. Med. Chem. Lett., 17, 1924 (2007); https://doi.org/10.1016/j.bmcl.2007.01.038.

34. K. Katano, E. Shitara, M. Shimizu, K. Sasaki, T. Miura, Y. Isomura, M. Kawaguchi, S. Ohuchi and T. Tsuruoka, Bioorg. Med. Chem. Lett., 6, 2601 (1996); https://doi.org/10.1016/0960-894X(96)00476-3.

35. A. Esanu, Thienopyridine Derivatives and Anti-thrombotic Compositions Containing the same US Patent 4,681,888 (1987).

36. H. Koike, F. Asai, A. Sugidachi, T. Kimura, T. Inoue, S. Nishino and Y. Tsuzaki, Tetrahydrothienopyridine Derivatives, Furo and Pyrrolo Analogs Thereof and Their Preparation and Uses for Inhibiting Blood Platelet Aggregation, US Patent 5,288,726 (1994). 